\title{
Causas de óbito e razões para eutanásia em uma população hospitalar de cães e gatos
}

\author{
Causes of death and reasons for euthanasia in a hospital population of dogs and cats
}

\author{
Sílvia Manduca TRAPP ${ }^{1}$; André Iurchiag IACUZIO ${ }^{1}$; Flávio Antonio BARCA JUNIOR ${ }^{1}$; Bernardo \\ KEMPER $^{1}$; Luiz César da SILVA ${ }^{1}$; Werner OKANO $^{1}$; Neide Mariko TANAKA ${ }^{1}$; Fabíola Cristine de \\ Almeida Rêgo GRECCO ${ }^{1}$; Luiz Fernando Coelho da CUNHA FILHO ${ }^{1}$; Fabiana de Andrade Melo \\ STERZA $^{1}$
}

${ }^{1}$ Curso de Medicina Veterinária da Universidade Norte do Paraná, Arapongas-PR

\begin{abstract}
Resumo
Com o objetivo de determinar as principais causas de morte em cães e gatos, foram analisadas informações correspondentes às fichas clínicas de cães e gatos provenientes de um hospital veterinário universitário entre julho de 2005 e julho de 2009. Durante o período estudado foram atendidos 2243 casos novos, sendo 2075 cães e 168 gatos. Em cães, o percentual de óbito foi de 10,6\% (220/2075) e em gatos, 8,92\% (15/168). A idade média quando do óbito em cães e gatos foi de 59,97 e 82,79 meses, respectivamente. Em cães, as principais causas de morte e razões para eutanásia foram distúrbios infecciosos ou parasitários, distúrbios causados por agentes físicos e neoplasias. Exceto pela idade, as demais características de resenha não influenciaram a expectativa de vida ou a causa de morte em cães. Em gatos, distúrbios provocados por agentes físicos e distúrbios urinários foram os principais motivos que resultaram na morte do animal. Os resultados observados identificam a necessidade de medidas profiláticas que possibilitarão maior expectativa de vida e, consequentemente, alteração na frequência das principais causas de morte e razões para eutanásia em cães e gatos.
\end{abstract}

Palavras-chave: Mortalidade. Motivo. Distúrbios infecciosos e parasitários. Agentes físicos.

\begin{abstract}
To determine the main causes of death in a population of dogs and cats from a University Veterinary Hospital, data from clinical records obtained from July, 2005 to July, 2009 were analyzed. During that period, 2,243 animals were taken to the hospital, 2,075 dogs and 168 cats. The percentage of death was $10.6 \%(220 / 2.075)$ in dogs, and 8.92\% (15/168) in cats. The median age at death was 59.97 months for dogs and 82.79 months for cats. The main causes of death and reasons for euthanasia in dogs were infectious or parasitic diseases, diseases caused by physical agents and neoplasia. Except by their age, all other characteristics described did not have an effect on life expectancy or death causes in dogs. In cats, physical agents and urinary diseases were the main cause of mortality. The results observed in this work identify the need for prophylactic measures that will enable a better life expectancy, and consequently, change the frequency of the main causes of death and reasons for euthanasia in dogs and cats.
\end{abstract}

Keywords: Mortality. Motive. Infectious or parasitic diseases. Physical agents.

\section{Introdução}

A alteração do panorama epidemiológico quanto à morbidade e mortalidade em cães e gatos é consequente, em parte, das condições sócio-econômicas da população, assim como da expectativa de vida desses animais, uma vez que se tem observado maior longevidade em cães e gatos. Considerando a melhoria desses aspectos, há uma tendência na redução da incidência de doenças infecto-contagiosas, altamente prevalentes em filhotes, e aumento da expressão de outras doenças associadas à senilidade ${ }^{1}$. Relatos in- ternacionais já têm evidenciado outros distúrbios que não os infecciosos como principais causas de óbitos em cães, dentre eles as doenças neurológicas, cardíacas e neoplásicas ${ }^{2}$.

\section{Correspondência para:}

Sílvia Manduca Trapp

Rua Acre, n. 18, Jd. Primavera, Jaguapitã - PR

CEP.: 86610-000

Tel.: 43-88065115

E-mail: smanducatrapp@gmail.com

Recebido: 19/08/2009

Aprovado: 02/09/2010 
No Brasil, ainda são escassas as informações a respeito das principais causas de morte em cães e inexistentes em gatos. Na literatura consultada, foram encontrados somente dois trabalhos recentes que identificaram as principais causas de morte em cães, um na área metropolitana de São Paulo e outro na Mesorregião do Centro Ocidental Rio-Grandense, os quais evidenciaram ainda o predomínio das doenças infecciosas como a principal causa de morte ${ }^{3,4}$. Entretanto, em nossa região não há estudos semelhantes.

É relevante conhecer as principais causas de óbito em cães e gatos para o fornecimento de subsídios para o monitoramento, planejamento e avaliação das ações profiláticas, com o intuito de reduzir o percentual de óbito, assim como contribuir para a melhoria da qualidade de vida do animal e consequentemente de seu proprietário. A relação entre as características de resenha e a causa de morte também pode sugerir teorias sobre a etiologia de doenças e até mesmo permitir estudar determinadas populações animais como modelos para doenças humanas ${ }^{2}$.

Com base no fato de que na região estudada não há informações de parâmetros epidemiológicos sobre mortalidade em cães e gatos, esse estudo teve como objetivo determinar a prevalência das doenças ou distúrbios que culminam em morte ou que fazem com que os cães e gatos sejam submetidos à eutanásia.

\section{Material e Método}

Foram revisadas todas as fichas clínicas de cães e gatos, registradas em um hospital veterinário universitário - UNOPAR situado na cidade de Arapongas$\mathrm{PR}$, que morreram entre o período de julho de 2005 e julho de 2009. Os dados foram obtidos por meio da pesquisa dos prontuários e compilados em tabelas. Foi considerada a causa de morte documentada nos prontuários pelo médico veterinário responsável. Semelhante a outros estudos, foi estabelecido um único diagnóstico para cada caso ${ }^{1,2,3,5}$. Para todos os animais, foram pesquisados dados sobre a espécie, idade na data de ocorrência do óbito, sexo e porte.

Os distúrbios que causaram a morte espontânea (morte natural) ou que foram responsáveis pela eutanásia foram agrupados em: distúrbios causados por agentes físicos (traumas e obstruções gastrintestinais), distúrbios infecciosos ou parasitários, doenças degenerativas, neoplasias, doenças metabólicas e endocrinológicas, distúrbios iatrogênicos e intoxicações. As doenças que não foram classificadas em nenhum desses tópicos foram agrupadas sob a expressão "outros distúrbios", semelhante a outros estudos ${ }^{3,6}$. Os animais submetidos à eutanásia sem apresentar uma condição clínica que justificasse tal procedimento foram incluídos em um grupo denominado "eutanásia por conveniência" e aqueles que não tiveram um diagnóstico definitivo estabelecido foram inseridos no grupo de "distúrbios inconclusivos".

Os cães foram distribuídos em três grupos etários: filhote (idade inferior a um ano), adulto (idade maior ou igual a um e inferior a dez anos) e idoso (idade maior ou igual a dez anos). Quanto aos gatos, esses foram considerados idosos somente quando a idade foi igual ou superior a onze anos ${ }^{7}$. Em relação ao sexo, os animais foram considerados apenas como machos ou fêmeas, independente de serem castrados ou não. Independentemente da raça ou idade, os animais foram classificados segundo seu porte físico, levando-se em consideração seu peso em quilogramas, em porte pequeno (para animais com peso igual ou inferior a $9 \mathrm{~kg}$ ); porte médio (peso corpóreo entre 9,5 e $23 \mathrm{~kg}$ ); porte grande (peso corpóreo entre 23,5 e $40 \mathrm{~kg}$ ); e porte gigante (peso corpóreo superior a 40,5 kg), segundo a classificação proposta por Goldston e Hoskins ${ }^{7}$.

Para a metodologia de cálculos foi utilizado o teste de qui-quadrado $\left(\chi^{2}\right)$ para as proporções esperadas iguais ou maiores que 5, para valores menores utilizou-se o teste Exato de Fisher, ambos com nível mínimo de significância de 5\%. Em virtude da ausência de informações a respeito do perfil da população 
atendida durante o período de estudo, o número total de óbitos foi utilizado como parâmetro da população. Para a realização de cálculos referentes à idade dos animais somente foram contabilizados aqueles cujas fichas clínicas apresentaram a informação da idade exata quando do óbito.

\section{Resultados}

Dentre o período estudado foram atendidos 2243 casos novos, sendo 2075 cães e 168 gatos. Para cada uma das espécies canina e felina, o percentual de óbito foi de $10,6 \%(220 / 2075)$ e $8,9 \%(15 / 168)$, respectivamente. Houve distribuição uniforme dos dados de acordo com o gênero e a raça e não houve diferença significativa entre essas características e as causas que levaram a óbito (Tabela 1). Apesar do maior número de óbitos em cães de pequeno porte, a diversidade do porte e das causas de óbito levou a uma pulverização dos dados que impediram uma inferência precisa das informações (Tabela 1).

De acordo com a data do óbito, a longevidade para cães e gatos foi de 59,97 e 82,79 meses, respectivamente (Tabela 2). Em cães, a metade dos óbitos ocorreu até 36 meses de idade, sendo que $36,12 \%$ do total veio a óbito até o décimo mês de vida enquanto a outra metade teve o último registro de óbito aos 288 meses de idade (Tabela 2 e Figura 1). A infecção pelo parvovírus associada ou não a infecção pelo coronavírus foi responsável pelo óbito da maioria dos cães com até dez meses de idade.

Ao comparar a faixa etária canina, em filhotes o óbito ocorreu principalmente em decorrência de distúrbios infecciosos e parasitários ( $\mathrm{p}<0,0001)$, em adultos, em virtude de doenças infecciosas ou parasitárias, seguido por distúrbios causados por agentes físicos ( $\mathrm{p}<0,0001)$ e em idosos, por neoplasia ( $<<0,0001)$. De acordo com a causa do óbito, as mortes ocorridas por doenças infecciosas ou parasitárias foram significativamente superiores em filhotes ( $p<0,0001$ ), as ocorridas por distúrbios causados por agentes físicos foram mais frequentes em adultos $(p<0,0133)$ e as consequentes de neoplasias foram mais frequentes em idosos $(\mathrm{p}<0,0001)$.

Nesse estudo, as principais causas de morte observadas em cães foram distribuídas em relação à categoria de distúrbio diagnosticado, da seguinte forma, em ordem decrescente de prevalência: distúrbios infecciosos ou parasitários (47,27\%), distúrbios causados por agentes físicos (13,18\%), neoplasias (10\%),

Tabela 1 - Número de óbitos em cães de acordo com a causa do óbito, gênero, raça e porte. Arapongas - PR - julho de 2005 a julho de 2009

\begin{tabular}{|c|c|c|c|c|c|c|c|c|c|}
\hline \multirow{3}{*}{ DOENÇA / DISTÚRBIO } & \multicolumn{2}{|c|}{ Gênero } & \multicolumn{2}{|c|}{ Raça } & \multicolumn{5}{|c|}{ Porte (kg) } \\
\hline & \multirow{2}{*}{$\mathrm{M}$} & \multirow{2}{*}{$\mathrm{F}$} & \multirow{2}{*}{ Pura } & \multirow{2}{*}{ SRD } & \multirow{2}{*}{$\begin{array}{c}\leq 9,0 \\
\text { Pequeno }\end{array}$} & \multirow{2}{*}{$\begin{array}{l}\text { 9,5-23 } \\
\text { Médio }\end{array}$} & \multirow{2}{*}{$\begin{array}{l}23,5-40 \\
\text { Grande }\end{array}$} & \multirow{2}{*}{$\begin{array}{c}>40,5 \\
\text { Gigante }\end{array}$} & \multirow[t]{2}{*}{$\mathrm{ND}$} \\
\hline & & & & & & & & & \\
\hline Doenças infecciosas ou parasitárias & 59 & 45 & 48 & 56 & 70 & 22 & 5 & 0 & 7 \\
\hline Distúrbios causados por agentes físicos & 13 & 16 & 11 & 18 & 20 & 5 & 2 & 0 & 2 \\
\hline Neoplasias & 9 & 13 & 12 & 10 & 4 & 8 & 7 & 1 & 2 \\
\hline Doenças degenerativas & 10 & 9 & 13 & 6 & 5 & 11 & 3 & 0 & 0 \\
\hline Inconclusivos & 10 & 9 & 9 & 10 & 9 & 3 & 2 & 0 & 5 \\
\hline Outros distúrbios & 3 & 6 & 3 & 6 & 1 & 5 & 1 & 1 & 1 \\
\hline Eutanásia por conveniência & 4 & 3 & 3 & 4 & 3 & 1 & 1 & 0 & 2 \\
\hline Doenças metabólicas e endocrinológicas & 1 & 4 & 3 & 2 & 2 & 2 & 1 & 0 & 0 \\
\hline Distúrbios iatrogênicos & 2 & 1 & 1 & 2 & 1 & 2 & 0 & 0 & 0 \\
\hline Intoxicações & 1 & 2 & 2 & 1 & 1 & 0 & 1 & 1 & 0 \\
\hline Total & 112 & 108 & 105 & 115 & 116 & 59 & 23 & 3 & 19 \\
\hline
\end{tabular}

M: macho; F: fêmea; SRD: sem raça definida; ND: porte não determinado 
Tabela 2 - Medidas de posição e dispersão referentes a idade (meses) quando do óbito de cães e gatos atendidos em uma população hospitalar. Arapongas - PR - julho de 2005 a julho de 2009

\begin{tabular}{ccc}
\hline Parâmetro & Cão & Gato \\
\hline Média & 59,97 & 82,79 \\
Mediana & 36 & 96 \\
Moda & 60 & 156 \\
Desvio padrão & 62,17 & 69,78 \\
Quartil 1 & 5 & 11,25 \\
Quartil 3 & 96 & 138 \\
Intervalo & 287 & 200 \\
Mínimo & 1 & 4 \\
Máximo & 288 & 204 \\
Contagem & 191 & 14 \\
\hline
\end{tabular}

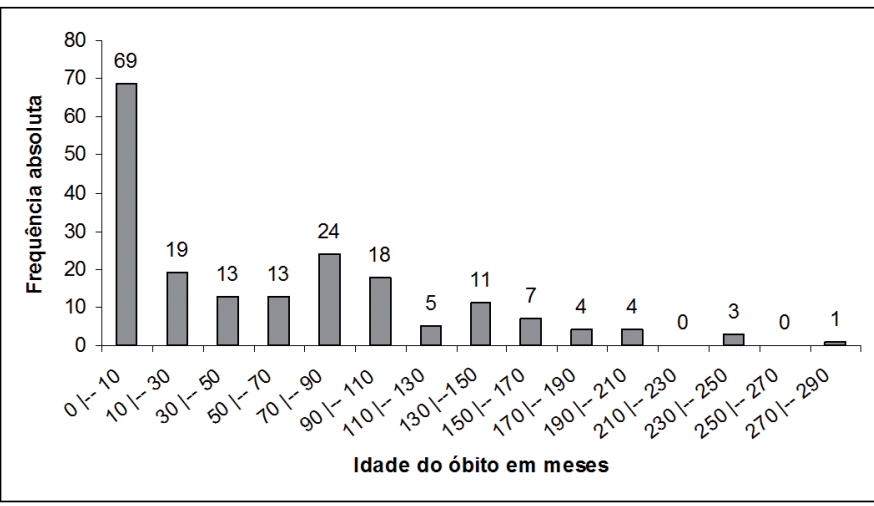

Figura 1 - Frequência absoluta de cães de acordo com a idade do óbito. Arapongas - PR - julho de 2005 a julho de 2009

doenças degenerativas $(8,63 \%)$, distúrbios inconclusivos (sem diagnóstico estabelecido) (8,63\%), outros distúrbios $(4,1 \%)$, eutanásia por conveniência $(3,18 \%)$, doenças metabólicas e endocrinológicas $(2,27 \%)$, distúrbios iatrogênicos $(1,37 \%)$ e intoxicações $(1,37 \%)$ (Tabela 3$)$.

Grande parte dos cães (104/220) morreu ou foi submetida à eutanásia consequente de doenças infecciosas ou parasitárias. Dentre essas, a cinomose e a parvovirose, associada ou não a coronavirose, foram as afecções mais frequentes, 49,03\% (51/104) e 42,03\% (44/104), respectivamente. Considerando a faixa etá- ria quando do óbito, $62,74 \%$ (32/51) dos cães com cinomose apresentaram entre um e nove anos de idade, enquanto que $97,72 \%$ (43/44) dos cães com parvovirose apresentaram idade inferior a um ano.

Do total de cães, 13,18\% (29/220) morreram ou foram submetidos à eutanásia por apresentarem distúrbios causados por agentes físicos. Dentre os traumas, $72,41 \%(21 / 29)$ foram decorrentes de atropelamento por veículo automotivo, $17,24 \%$ (5/29) decorrentes de briga entre animais, 6,9\% (2/29) decorrentes de obstrução gastrintestinal e 3,44\% (1/29) decorrentes de torção gástrica. 
Neoplasia foi a terceira causa mais frequente de óbito em cães, sendo que a eutanásia foi aplicada na maioria desses animais $(\mathrm{p}=0,0265)$ (Tabela 3). A idade média desses cães quando do óbito foi de 125,4 meses, dessa forma $68,18 \%(15 / 22)$ desses eram idosos. Neoplasma mamário foi o principal distúrbio neoplásico que culminou com a morte de 40,9 \% (9/22) dos cães.

Dentre as doenças degenerativas, a doença do disco intervertebral (DDIV) foi a causa mais frequente de óbito em cães $(36,84 \%$; $7 / 19)$ seguida por insuficiência cardíaca $(26,31 \% ; 5 / 19)$, insuficiência hepática $(21,05 \% ; 4 / 19)$, insuficiência renal $(10,52 \% ; 2 / 19)$ e displasia coxofemoral $(5,26 \% ; 1 / 19)$. A eutanásia foi aplicada em $63,15 \%$ (12/19) de todos os casos de doença degenerativa e em 100\% (7/7) dos casos de DDIV. Todos os pacientes com DDIV submetidos à eutanásia apresentaram grau $\mathrm{V}$ de disfunção e tinham indicação cirúrgica. Óbito em decorrência de doenças degenerativas foi significativamente mais frequente em cães adultos e idosos, $\mathrm{p}=0,0034$ e $\mathrm{p}=0,0002$, respectivamente, sendo a idade média quando do óbito de 109 meses.
Dentre as causas de óbito classificadas como outros distúrbios, sepse ocorreu em 77,77\% (7/9) dos cães, seguido por um óbito decorrente de complicações em partos distócicos e outro decorrente de pancreatite aguda. Os casos de sepse foram classificados conforme o diagnóstico estabelecido na ficha clínica hospitalar pelo veterinário responsável pelo atendimento, que considerou os sinais clínicos e laboratoriais para tal, não sendo relacionada a causa responsável pela sepse.

Como evidenciado na tabela 3, 8,63\% (19/220) dos óbitos em cães foram classificados como inconclusivos por não apresentarem um diagnóstico definitivo estabelecido. Doença crônica envolvendo o tegumento foi responsável pela aplicação da eutanásia por conveniência em cinco dos sete casos de óbito. Piometra $(4 / 5)$ e cetoacidose diabética (1/5) foram as causas de óbito classificadas como doenças metabólicas e endocrinológicas. Depressão anestésica (1/3), hemorragia pós-operatória $(1 / 3)$ e úlcera gástrica secundária ao uso de antiinflamatório não esteroidal (1/3) foram os motivos de óbitos classificados como iatrogênicos. Entre os óbitos resultantes de quadros de intoxicação,

Tabela 3 - Número e frequência de óbitos em cães de acordo com a causa e a natureza da morte. Arapongas - PR - julho de 2005 a julho de 2009

\begin{tabular}{lcccc}
\hline \multicolumn{1}{c}{ DOENÇA / DISTÚRBIO } & Eutanásia & Natural & Total & Frequência \\
\hline & & & $\%$ \\
Doenças infecciosas ou parasitárias & 44 & 60 & 104 & 47,27 \\
Distúrbios causados por agentes físicos & 18 & 11 & 29 & 13,18 \\
Neoplasias & 17 & 5 & 22 & 10 \\
Doenças degenerativas & 12 & 7 & 19 & 8,63 \\
Inconclusivos & 7 & 12 & 19 & 8,63 \\
Outros distúrbios & 2 & 7 & 9 & 4,1 \\
Eutanásia por conveniência & 7 & 0 & 7 & 3,18 \\
Doenças metabólicas e endocrinológicas & 3 & 2 & 5 & 2,27 \\
Distúrbios iatrogênicos & 0 & 3 & 3 & 1,37 \\
Intoxicações & 0 & 3 & 3 & 1,37 \\
\hline Total & 110 & 110 & 220 & 100 \\
\hline
\end{tabular}


foram identificados o anticolinesterásico carbamato e anticoagulante cumarínico varfarina.

Quanto aos gatos, as principais causas de morte foram distúrbios causados por agentes físicos (4/15), distúrbios urinários (4/15), neoplasias (3/15), outros distúrbios (2/15), eutanásia por conveniência (1/15) e intoxicação (1/15). As causas de óbito prevalentes em relação aos distúrbios causados por agentes físicos foram traumas decorrentes de atropelamento e agressão por cães, quanto aos distúrbios urinários, todos os gatos morreram em virtude de complicações da doença idiopática do trato urinário inferior dos felinos.

\section{Discussão}

Não foi possível comparar o percentual de óbito na população estudada, uma vez que as pesquisas, geralmente, avaliam registros de necropsia junto a laboratórios de patologia, registros oriundos de cemitérios de animais de companhia ou de companhias de seguro e informações fornecidas por proprietários de clínicas veterinárias, canis ou gatis ${ }^{2,3,4,8,9}$.

De acordo com Bronson ${ }^{10}$, o gênero não influenciou a sobrevivência dos animais. Entretanto, Bentubo et al. ${ }^{4}$ observaram maior longevidade das fêmeas em relação aos machos, sugerindo esse fato consequente do hábito de cães machos abandonarem suas residências em busca de acasalamento, estando mais expostos às condições ambientais que colocam as suas vidas em risco; por sua vez, as fêmeas permanecem mais tempo isoladas na época de puerpério ${ }^{11}$. Quanto ao porte dos animais, Goldston e Hoskins ${ }^{7}$ relataram que espécies com alta frequência cardíaca apresentam menor sobrevivência. Nesse sentido, Bentubo et al. $^{4}$ demonstraram que há influência do tamanho dos cães de diferentes raças na expectativa de vida, uma vez que os animais de pequeno porte sobreviveram menos que os animais de porte médio, grande e gigante contradizendo Deeb e Wolf ${ }^{12}$ que relataram maior sobrevivência em cães de pequeno porte. Mesmo observando um número maior de óbitos em cães de pequeno porte, não nos foi possível analisar a importância deste fato por não haver um estudo do perfil da população atendida no local estudado.

$\mathrm{Na}$ literatura consultada não foram encontradas informações a respeito da expectativa de vida em gatos. Quanto aos cães, houve discrepância entre os resultados relatados, conforme o país de origem das pesquisas. Estudos realizados na Dinamarca ${ }^{6}$, na Inglaterra ${ }^{13}$ e nos Estados Unidos ${ }^{10}$ têm evidenciado idade de sobrevida entre 117 e 132 meses. No Brasil, a avaliação da população de cães da área metropolitana de São Paulo identificou a idade média de 36 meses quando do óbito. Assim, a longevidade canina aqui apresentada foi superior a nacional e inferior a internacional. Contudo, é questionável a representatividade desses valores para a população, uma vez que os óbitos não apresentaram distribuição normal.

Em muitos países, o câncer é mencionado como a principal causa de morte canina cuja prevalência como causa de óbito ou razão para eutanásia é de 14 a $39,5 \% \%^{2,5,6,10,14}$. A frequência do óbito em cães por neoplasma aqui observada se aproxima a relatos nacionais que oscilam entre 7,8\% e 13,3\% ${ }^{4}$. Possivelmente, a diferença observada entre os países seja decorrente da menor expectativa de vida dos cães no Brasil.

Dentre os neoplasmas, o mamário foi o mais ocorrente. $\mathrm{O}$ tumor em glândulas mamárias é considerado o mais frequente em cadelas, correspondendo a $70 \%$ dos tumores diagnosticados nesses animais ${ }^{15}$.

Semelhante aos demais estudos conduzidos no Brasil, as doenças infecciosas ou parasitárias destacaramse como a causa mais comum de óbitos em cães, tratando-se de um fator de risco bem caracterizado, que reduz a expectativa de vida ${ }^{3,4}$. No entanto, em outros países, é baixa a prevalência de óbito consequente de doenças infecciosas ou parasitárias ${ }^{2,5,6,14}$.

Acredita-se que a alta frequência das doenças infecciosas ou parasitárias como causa de morte em cães no Brasil esteja relacionada à baixa adesão aos progra- 
mas de vacinação e everminação ${ }^{3}$. Medidas profiláticas como imunoprofilaxia são fundamentais para o controle de doenças infecciosas, contudo, a aplicação dessas esbarra, possivelmente, na falta de conhecimento, aspectos sócio-econômicos e negligência dos proprietários ${ }^{4}$.

Como evidenciado, distúrbio provocado por agentes físicos foi a segunda causa mais importante de óbito em cães. Comparado a relatos nacionais e internacionais, mortes por traumatismos se enquadram entre as três principais causas de morte em cães $^{2,3,4}$. A maioria desses animais morreu em decorrência de atropelamento por veículo automotivo sugerindo insuficiente prática da posse responsável por parte dos proprietários. Dessa forma, são necessárias orientações, por parte dos médicos veterinários, quanto à adoção de medidas de segurança que minimizem tais riscos ${ }^{2,4}$.

A sepse foi a principal responsável por mortes na categoria outros distúrbios. Essa é uma crescente causa de morte, tendo como fatores contribuintes para o seu surgimento o emprego de técnicas invasivas, imunossupressão e infecções hospitalares. A precocidade do reconhecimento da sepse parece ser a forma mais provável de reduzir a mortalidade ${ }^{16}$.

Do total dos casos, sete cães foram submetidos à eutanásia por conveniência. Cinco desses, a eutanásia foi motivada por lesões tegumentares, das quais a maioria era crônica. De acordo com Fighera et al. ${ }^{3}$, lesões traumáticas com possibilidade de tratamento clínico-cirúrgico, lesões crônicas de pele, motivo fútil (não associado à doença) e neoplasmas benignos são situações associadas à eutanásia sem justificativa clínica em cães. Esses autores relataram 2,1\% (101/4844) dos cães submetidos à eutanásia por conveniência.

Distúrbios iatrogênicos perfizeram 1,37\% de todas as mortes neste estudo, sendo depressão anestésica, hemorragia pós-cirúrgica e ulceração gástrica as causas dos óbitos. Fighera et al. ${ }^{3}$ relataram resultados iguais aos aqui apresentados, no entanto os demais trabalhos não consideraram os transtornos de origem iatrogênica como causa de morte.

Quanto ao óbito resultante de doenças degenerativas, os resultados aqui apresentados foram parecidos aos de Fighera et al. ${ }^{3}$, que relataram a prevalência dessa causa de morte em 7,1\% dos cães necropsiados. Dentre as doenças degenerativas, doença do disco intervertebral e insuficiência cardíaca foram as mais observadas como responsáveis por óbitos em cães, os quais apresentaram idade média ao óbito de 109 meses. Olsen e Allen ${ }^{8}$ ao examinarem registros de necropsias em cães, identificaram a doença cardíaca como a principal causa de morte súbita em cães. Bonnett et al. ${ }^{2}$ ao avaliarem registros de cães pertencentes a uma companhia de seguros na Suíça, cujos cães apresentavam idade maior ou igual a 120 meses, evidenciaram a insuficiência cardíaca como uma das principais causas de óbito.

Como observado, a eutanásia foi frequente em cães com DDIV. Em parte, essa condição pode refletir a indisponibilidade do proprietário em assumir os custos terapêuticos ou mesmo o compromisso quanto aos cuidados de enfermagem. O prognóstico reservado é outro fator influente na conduta terapêutica, entretanto, informações mais recentes têm evidenciado a recuperação satisfatória de animais com DDIV grau $\mathrm{V}$ submetidos à cirurgia descompressiva ${ }^{17}$. Dessa forma, perante esses novos resultados, espera-se que a estimativa quanto à evolução clínica do paciente seja mais otimista resultando em maior adesão a terapêutica cirúrgica e menor prática da eutanásia nesses pacientes.

Infelizmente, a comparação com os demais relatos é limitada em virtude da classificação diferencial dos distúrbios entre os autores, uma vez que algumas doenças são classificadas como doenças degenerativas e outras como doenças da senilidade ${ }^{4}$. Especificamente a doença do disco intervertebral, ora é classificada como doença degenerativa ${ }^{3}$ e ora classificada somente como doença neurológica ${ }^{2,4}$. Outros exemplos são os 
diagnósticos de insuficiência hepática ou renal, piometra e diabete melito, às vezes classificados como distúrbios metabólicos e endocrinológicos ${ }^{3}$ e outras vezes classificados como outros distúrbios ${ }^{2}$ ou mesmo em sistema urogenital, quando se trata do diagnóstico de piometra ${ }^{8}$.

Em virtude da escassez de informações a respeito das principais causas de óbito em cães e gatos, os resultados obtidos nesse trabalho são importantes para futuras comparações, para orientar na adoção de medidas profiláticas que valorizem a saúde animal e con-

\section{Referências}

1. BONNETT, B. N.; EGENVALL, A.; OLSON, P.; HEDHAMMAR, A. Mortality in insured Swedish dogs: rates and causes of death in various breeds. Veterinary Record, v. 141, n. 2, p. 40-44, 1997.

2. BONNETT, B. N.; EGENVALL, A.; HEDHAMMAR, A.; OLSON, P. Mortality in over 350,000 insured swedish dogs from 1995-2000: I. Breed-, gender-, age- and cause-specific rates. Acta Veterinaria Scandinavica, v. 46, n. 3, p. 105-120, 2005.

3. FIGHERA, R. A.; SOUZA, T. M.; BRUM, J. S.; GRACA, D. L.; KOMMERS, G. D.; IRIGOYEN, L. F.; BARROS, C. S. L. Causas de morte e razões para eutanásia de cães da Mesorregião do Centro Ocidental Rio-Grandense (1965-2004). Pesquisa Veterinária Brasileira, v. 28, n. 4, p. 223-230, 2008.

4. BENTUBO, H. D. L.; TOMAZ, M. A.; BONDAN, E. F.; LALLO, M. A. Expectativa de vida e causas de morte em cães na área metropolitana de São Paulo (Brasil). Ciência Rural, v. 37, n. 4, p. 1021-1026, 2007.

5. MOORE, G. E.; BURKMAN, K. D.; CARTER, M. N.; PETERSON, M. R. Causes of death or reasons for euthanasia in military working dogs: 927 cases (1993-1996). Journal of the American Veterinary Medical Association, v. 219, p. 209$214,2001$.

6. PROSCHOWSKY, H. F.; RUGBJERG, H.; ERSBOLL, A. K. Mortality of purebreed and mixed-breed dogs in Denmark. Preventive Veterinary Medicine, v. 58, n. 2, p. 63-74, 2003.

7. GOLDSTON, R. T.; HOSKINS, J. D. Geriatria e gerontologia do cão e do gato. São Paulo: Roca, 1999. 551 p.

8. OLSEN, T. F.; ALLEN, A. L. Causes of sudden and unexpected death in dogs: a 10-year retrospective study. The Canadian Veterinary Journal, v. 41, n. 11, p. 873-875, 2000.

9. HAYASHIDANI, H.; OMI, Y.; OGAWA, M.; FUKUTOMI, K. Epidemiological studies on the expectation of life for dogs computed from animal cemetery records. Japanese Journal Veterinary Science, v. 50, n. 5, p. 1003-1008, 1988.

10.BRONSON, R. T. Variation in age at death of dogs of different sequentemente de seu proprietário e até mesmo para aumentar a longevidade do animal.

\section{Conclusões}

Este estudo permite concluir que as principais causas de morte em cães foram distúrbios infecciosos ou parasitários, distúrbios causados por agentes físicos e neoplasias, enquanto em gatos foram distúrbios causados por agentes físicos e distúrbios urinários. Os resultados evidenciados refletem a necessidade de maior adesão aos programas de imunoprofilaxia e prática da posse responsável.

sexes and breeds. American Journal of Veterinary Research, v. 43 , n. 1 , p. $2057-2059,1982$.

11.BERZINS, M. A. V. S. Velhos, cães e gatos: interpretação de uma relação. 2000. 132 f. Dissertação (Mestrado em Gerontologia) - Pontifícia Universidade Católica de São Paulo, São Paulo, 2000.

12.DEEB, B. J.; WOLF, N. S. Studying longevity and morbidity in giant and small breeds of dogs. Veterinary Medicine, v. 89, p. 702-713, 1994. Supplement, 7.

13. MICHELL, A. R. Longevity of british breeds of dogs and its relationships with sex, size, cardiovascular variables and disease. Veterinary Record, v. 145, n. 27, p. 625-629, 1999.

14.CRAIG, L. E. Cause of death in dogs according to breed: a necropsy survey of five breeds. Journal of the American Animal Hospital Association, v. 37, p. 438-443, 2001.

15. MERLO, D. F.; ROSSI, L.; PELLEGRINO, C.; CEPPI, M.; CARDELLINO, U.; CAPURRO, C.; RATTO, A.; SAMPUCCO, P. L.; SESTITO, V.; TANARA, G.; BOCCHINI, V. Cancer incidence in pet dogs: findings of the Animal Tumor Registry of Genoa, Italy. Journal of Veterinary Internal Medicine, v. 22, n. 4, p. 976-984, 2008

16.DELLINGER, R. P.; LEVY, M. M.; CARLET, J. M.; BION, J.; PARKER, M. M.; JAESCHKE, R.; REINHART, K.; ANGUS, D. C.; BRUN-BUISSON, C.; BEALE, R.; CALANDRA, T.; DHAINAUT, J. F.; GERLACH, H.; HARVEY, M.; MARINI, J. J.; MARSHALL, J.; RANIERI, M.; RAMSAY, G.; SEVRANSKY, J.; THOMPSON, B. T.; TOWNSEND, S.; VENDER, J. S.; ZIMMERMAN, J. L.; VINCENT, J. L. Surviving sepsis campaign: international guidelines for management of severe sepsis and septic shock: 2008. Intensive Care Medicine, v. 34, n. 4 , p. $783-785,2008$.

17. ARIAS, M. V. B.; NISHIOKA, C. M.; GARCIA, C. O.; REIA, A. Z.; BARAUNA JUNIOR, D.; MARCASSO, R. A. Avaliação dos resultados clínicos após cirúrgia descompressiva em cães com doença do disco intervertebral. Arquivo Brasileiro de Medicina Veterinária e Zootecnia, v. 59, n. 6, p. 1445-1450, 2007. 